\title{
Research on Aircraft Surface Taxi Path Planning and Conflict Detection and Resolution
}

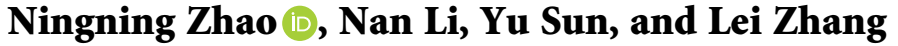 \\ Key Laboratory of Civil Aviation Flight Wide-Area Surveillance and Safety Control Technology, \\ School of Air Traffic Management, CAUC, Tianjin 300300, China \\ Correspondence should be addressed to Ningning Zhao; xianyuer315@163.com
}

Received 30 March 2021; Revised 24 July 2021; Accepted 4 September 2021; Published 5 October 2021

Academic Editor: Rui Jiang

Copyright $(92021$ Ningning Zhao et al. This is an open access article distributed under the Creative Commons Attribution License, which permits unrestricted use, distribution, and reproduction in any medium, provided the original work is properly cited.

Aircraft surface taxiing conflict avoidance is mostly adopted by waiting and rerouting methods, but this method does not consider the difference in total taxiing time that may be caused by different strategies. In this study, the airport taxiing path optimization model and taxiing area division model are constructed first. Then, the taxiway use is controlled by subregion based on the analysis of the surface activity area connection relationship. Based on the results of aircraft surface taxiway preselection, the time window of the taxiing area is solved and conflict detection is performed. For aircraft with taxiing conflicts, waiting or changing paths is selected to deconflict taxiing by comparing priorities. An improved $A *$ algorithm solution is applied to generate conflict-free glide paths and new glide trajectory occupancy time windows, while the glide paths of aircraft without glide conflicts are not affected. The results of the study show that the use of time windows for conflict detection and deconfliction can further reduce the total taxiing time of aircraft operating on the surface, resulting in a significant reduction in the number of aircraft conflicts, and thus, airport operational safety is ensured. This study has a high practical value and is expected to be applied in the real-time control decision of aircraft taxiing in the future.

\section{Introduction}

With the continuous development of the goal of "building a powerful air transport country," my country's civil aviation industry has developed rapidly in recent years. According to statistics, the compound annual growth rates of my country's total civil aviation transportation turnover, civil aviation passenger transportation and turnover, and civil aviation cargo and mail transportation and turnover from 2011 to 2018 were $11.10 \%, 11.08 \%, 13.06 \%, 4.10 \%, 4.10 \%$, and $6.06 \%$, respectively [1]. With the increase in flight business, major airports have been constantly renovating and expanding to cope with the increasingly congested traffic volume. At the same time, various airports across the country have also adopted measures such as increasing runways and parking spaces, building more terminal buildings, and constantly introducing field monitoring equipment. Other measures are to further increase the surface operating capacity. However, as the number of multirunway airports continues to increase, the airport surface structure is becoming increasingly complex. As the multirunway system is in operation, the flight activities between the various runways and the airport airspace affect each other, which further causes the problem of surface conflict and congestion to become more serious. According to statistics, the average delay time for departures from domestic airports in 2019 is about 28.16 minutes, of which the average delay time for departure in July reached 39.51 minutes [2]. According to statistics, the direct economic loss caused by every $10 \%$ of flight delays is as high as 1.5 billion euros [3]. The increasingly complicated airport scene layout has led to frequent taxiing congestion incidents on the scene, threatening the safety of aircraft taxiing and greatly reducing the efficiency of airport operations. Literature 4 statistics on the average taxi time of flights at major airports across the country in 2018 show that Urumqi Diwopu Airport has the longest taxi time at 27.8 minutes and Beijing Capital Airport has an average taxi time of about 25.3 minutes, ranking second. The shortest taxi time is Sanya 
Phoenix Airport, which is 13.4 minutes. In 2018, the average extra taxi time data of my country's airports was about 1.5 minutes per sortie, which is similar to Europe. However, some busy airports in my country have the problem of more extra taxi time, with an average of about 4.94 minutes per sortie, while the throughput of Europe is relatively high. The extra taxi time of a large airport is $2.8 \mathrm{~min} /$ sort. The problem of airport capacity restriction caused by the aircraft's long taxi time cannot be ignored [4]. Therefore, the conflict between the limited airport resources and the increasing passenger traffic volume in China is becoming increasingly serious, and the consequent problems, including airport surface conflict and declining of flight punctuality, are also becoming severer; thus, effective trajectory prediction and conflict resolution is an effective way to improve the flight punctuality and guarantee the safe operation of flights. There are many research studies on $4 \mathrm{D}$ trajectory prediction and surface conflict at home and abroad. Jun Chen et al. used aircraft surface taxiing velocity profiles to compute low-cost and environmental $4 \mathrm{D}$ trajectories by combining $4 \mathrm{D}$ trajectories and path scheduling together into a multiobjective optimization subject [5]. Wickramasinghe et al. quantified the optimization of $4 \mathrm{D}$ trajectories in relation to aircraft flight plans and showed that $4 \mathrm{D}$ trajectories can optimize the flight plans of aircraft [6]. Zhang et al. used linear programming to allocate the arrival time of the control points. The particle swarm optimization methods are used to optimize the speed of the distribution control points. They generate an improved and more accurate taxiing speed curve [7]. Yang obtained optimized 4D trajectory predictions using an isometric trajectory modeling method [8]. Wang et al. used a multiobjective immune optimization method to solve the Pareto optimum speed curve of the aircraft by establishing an aircraft motion model as well as a discrete taxiing speed model, and the results showed that the method can provide a variety of accurate taxiing velocity profiles for the surface aircraft [9]. Podgrski and Skorupski constructed a dynamic taxiing path choice model to describe airport surface congestion by using conflict points and calculated the conflict resolution of taxiing paths through a simulation model [10]. Li et al. constructed a model with the least taxiing time as the objective function and the typical conflict of aircraft taxiing as the constraint to solve the optimized aircraft taxiing path by using a genetic algorithm. The results showed that the method effectively avoids taxiing conflicts [11]. Wang constructed an airport structure model to optimize the aircraft surface taxiing paths conditional on the temporal and spatial distribution characteristics of airport conflicts. The results showed that the model can lead to a reduction in aircraft taxiing time and a decrease in the number of surface taxiing conflicts [12]. To further reduce the number of aircraft surface conflicts, Li et al. constructed a model and solved it with the $A *$ algorithm to obtain the optimized aircraft taxiing time by taking the airport taxiing rules, aircraft wake interval, and conflict avoidance restrictions as constraints and the surface conflict as minimization objective function [13]. However, there are few research studies on the combination of conflict detection and relief of aircraft taxiing paths.
Studies have shown that when taxiing conflicts on the airport surface, aircraft waiting or replanning the route are often used to avoid conflicts, but the selection of different strategies may cause the total taxi time to be different. Here, the use of taxiway is controlled in a subregion based on the analysis of the airport movement area connection relationship, and the time window occupied by the taxiing area is solved for conflict detection based on preliminary results of the taxiing path on the aircraft scene. For the aircraft with taxiing conflicts, by comparing the priority, the waiting, or changing, the path is selected to deconflict the taxiing conflicts, and then, the time window occupied by the conflict-free taxiing path and the new navigational trajectory is generated for the aircraft.

\section{Planning and Solving of Aircraft Taxiing Path on the Surface}

$P$ surface taxiing paths are generated for any aircraft $k$ in the aircraft set $F=\{f 1, f 2, \ldots, f n\}$. Among them, the preselected article $z$ taxiing path $\operatorname{Lkz}(1 \leq k \leq n, 1 \leq z \leq p)$ is composed of a group of ordered nodes $\{N 1, N 2, \ldots, N q\}$. There are $M$ nodes in the airport network model $V$; therefore, $\leq M, N_{i}, N_{j}, N_{r} \in V,\left(N_{i}, N_{j}\right) \in E, i=1,2, \ldots, q, j=1$, $2, \ldots, q$, among which the meanings of variables are as follows.

$x_{i j}=1$, which means that the aircraft $k$ taxis from node $N_{i}$ to node $N_{j}$; otherwise, $x_{i j}=0$;

$d_{i j}$ is the length of straight-line taxiway section $\left(N_{i}, N_{j}\right)$ in the taxiway system

$t_{i j}$ is the taxiing time of the aircraft on the straight-line taxiing section $\left(N_{i}, N_{j}\right)$

$R_{r}=1$ indicates that the aircraft $k$ turns at the node $N_{r}$; otherwise, $R_{r}=0$;

$d_{r}$ is the turning length at the node $N_{r}$ during aircraft surface taxiing

$t_{r}$ is the turning and taxiing time of the aircraft at the node $N_{r}$. Then, the total taxiing distance of the aircraft can be obtained from the following formula:

$$
D=\sum_{i=1}^{m} \sum_{j=1}^{m} x_{i j} d_{i j}+\sum_{i=1}^{m} R_{r} d_{r}
$$

There are two objective functions: the first $\sum_{i=1}^{m} \sum_{j=1}^{m} x_{i j} d_{i j}$ is the taxiing distance of the aircraft on each straight taxiing section. Among them, $x_{i j}$ is determined by the node $N_{i}$ taxiing straight to the node $N_{j}$ whether the aircraft $k$ is routed to the node $N_{j}$, the value is 1 or 0 , and $d_{i j}$ is the length of the taxiing road segment $\left(N_{i}, N_{j}\right)$; the second $\sum_{i=1}^{m} R_{r} d_{r}$ is the turning distance of the aircraft turning node, which depends on whether the aircraft is at the node $N_{r}$. Decide when you turn, $R_{i}$ takes the value 1 or 0 , and $d_{r}$ can be expressed as

$$
d_{r}=\frac{\theta_{r} \pi r}{180}
$$

The taxiing process of the aircraft turning section is shown in Figure 1. 


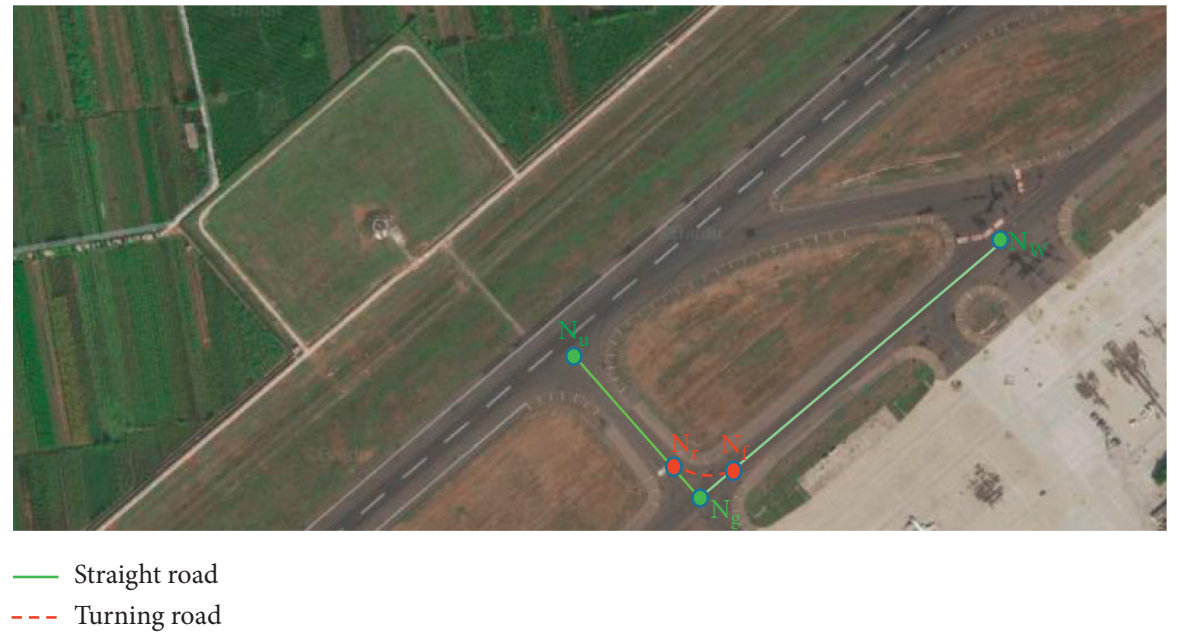

FIgURE 1: The taxiing process of the aircraft turning section.

As shown in Figure 1, the calculation assumes that the scene aircraft $k$ starts taxiing at the node $N_{u}$ and passes through the $N_{g}$ and $N_{w}$ nodes in turn. In reality, the aircraft has a turn length at the $N_{g}$ point, and the aircraft starts taxiing at the $N_{u}$ point, turning from the $N_{r}$ point. $N_{f}$ end the turn at a point and continue to slide to $N_{w}$. The actual length of the taxiing distance of the aircraft on this section is the sum of the straight length $d_{f w}$, the straight length $d_{u r}$, and the turning length $d_{r}$.

Because there are many variables in the model, the Bellman-Ford algorithm and $A *$ algorithm are used to solve the problem. The cost evaluation function of the $A *$ algorithm is

$$
f(n)=g(n)+h(n),
$$

where $n$ is the current node; $f(n)$ is the cost evaluation function from the starting point through the node $n$ to the target point; $g(n)$ is the actual cost from the starting node to the node $n$, and $h(n)$ is the estimated cost from the node $n$ to the target point. When $n$ is the starting node, $(n)=0$; if $h(n)=0$, the $A *$ algorithm is the Dijkstra algorithm without heuristic information. The search goal of the $A *$ algorithm is to find the $k$ path with the shortest taxi distance for the request aircraft; then, $f(n)$ is the total taxi distance from the starting node to the target node, and $g(n)$ is the glide distance from the initial node to the current node $n . h(n)$ is preprocessed before the algorithm is executed. Reverse all sections of the airport, execute the Bellman-Ford algorithm starting from the target point, and calculate the shortest sliding distance from the target point to all nodes in advance, and the calculated distance value is $h(n)$.

\section{Aircraft Taxiing Conflict Types and Time Windows}

3.1. Types of Aircraft Taxiing Conflicts. Aircraft taxiing conflicts at the surface are mainly of the following three types [14].
3.1.1. Head-to-Head Conflict. Assuming that aircraft 1 and aircraft 2 are expected to arrive at the same or similar times on the same taxiway, a head-on conflict will be caused when the two aircraft taxi in opposite directions, as shown in Figure 2.

To avoid head-to-head conflicts in the surface, taxiing, ATC can direct aircraft 2 to stop at intersection B for waiting according to the priority. If aircraft 2 has already passed intersection $B$, dynamic path planning will change the taxiing path of aircraft 1 and direct aircraft 1 to pass through intersection A without interrupting the taxiing of both aircraft 1 and aircraft 2 .

3.1.2. Cross-Conflict. Assuming that aircraft 1 and aircraft 2 pass through the same intersection and are expected to arrive at the intersection at the same or a similar time, a crossover conflict may arise, as shown in Figure 3.

To avoid surface taxi crossover conflicts, the ATC can instruct aircraft 2 to wait before reaching the intersection to avoid aircraft 1 , and after it passes, aircraft 2 continues taxiing on a preplanned trajectory while maintaining wake separation.

3.1.3. Rear-End Conflict. Assume that aircraft 1 and aircraft 2 are taxiing in the same direction with different taxiing speeds on the same taxi line. The distance between the rear aircraft and the front aircraft is less than the minimum safety interval, which may cause a rear-end conflict, as shown in Figure 4.

To avoid rear-end conflicts at the surface, the ATC may command aircraft 2 not to enter the section until aircraft 1 passes this section and meet the safe wake interval.

\subsection{Aircraft Taxiing Area and Taxi Time Window}

3.2.1. Taxiing Area Division Model. According to the airport network model, the taxiway area connection relationship is known, and then, the taxiway is divided into several nonoverlapping areas according to the area connection 


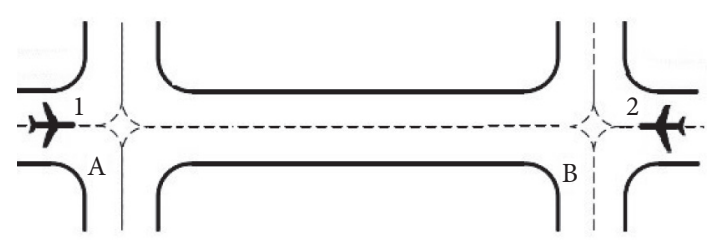

FIgURE 2: Head-to-head conflict.

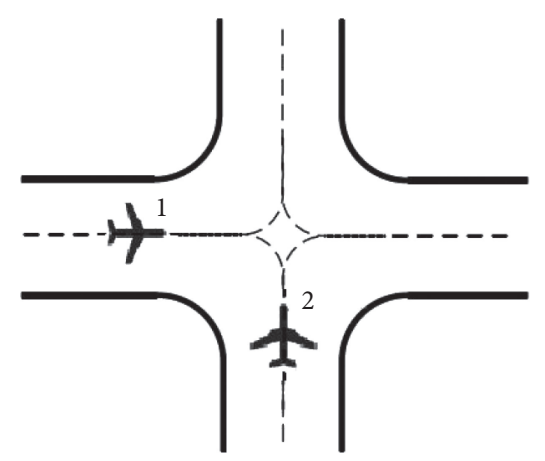

FIgURe 3: Cross-conflict.

relationship to obtain the taxiway area division model. Finally, the two are superimposed according to the spatial location to obtain the hierarchical taxiway structure model, as shown in Figure 5.

The safety of aircraft taxiing is ensured by the method of controlled operation of the surface in zones, limiting each zone to one aircraft. To improve taxiway utilization efficiency, longer paths are segmented based on node locations. The algorithm generates forecast data for the aircraft after receiving its request, including the aircraft taxiing path, the velocity profile in each road segment, the desired arrival time at each point, and the taxiing time window corresponding to each region. When the aircraft taxiing area occupancy time window conflict is detected, the conflict is deconflicted based on the current aircraft surface taxiway occupancy time window situation to meet the area usage restriction requirements. After the conflict resolution is completed, timely update the prediction path of the surface sliding and the time window of the area occupation time window to ensure that the predicted velocity profile corresponds to the regional occupation time window.

3.2.2. Aircraft Taxi Time Window. Based on the aircraft request information and taxi path prediction results, calculate the moments when the aircraft enters each taxiway area on the taxiing trajectory and leaves each taxiway area and then solve the occupation time window of each area on the taxiing trajectory $[15,16]$.

Assume that the moment when aircraft $k$ enters taxiing region $Q_{i}$ is denoted as $t_{i}^{\text {in }} k$ and the moment when it leaves taxiing region $Q_{i}$ is denoted as $t_{i}^{\text {out }} k$. If $Q_{i}$ is the starting taxiing area, the time when aircraft $k$ enters the area $Q_{i}$ is equal to the path planning start time $t_{0}$. If $Q_{i}$ is not the starting taxiing area, then aircraft $k$ entering the taxiing area $Q_{i}$ at the time $t_{i}^{\text {in }} k$ is the moment $t_{i-1}^{\text {out }} k$ of leaving the taxiing

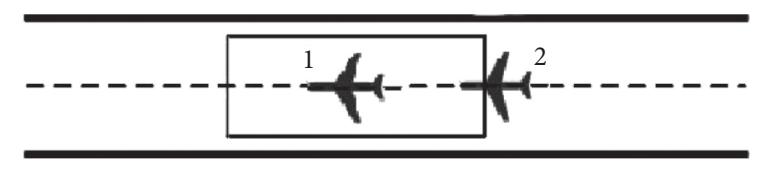

FIGURE 4: Rear-end conflict.

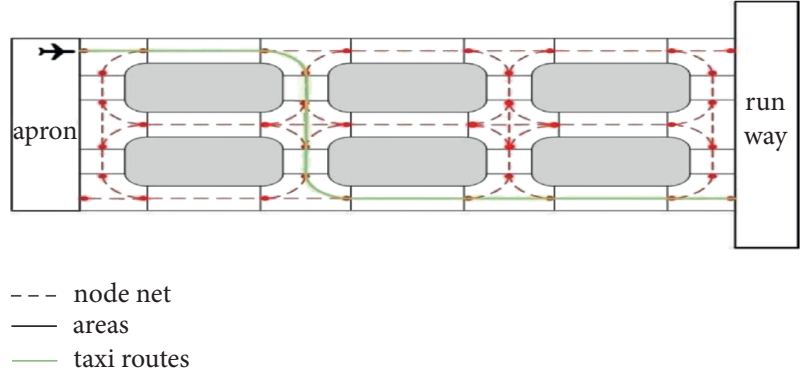

FIGURE 5: Schematic diagram of the taxiway node network, area division, and taxi path.

area $Q_{i-1}$. If $Q_{i}$ is the ending taxiing area, the time when the aircraft $k$ leaves the area $Q_{i}, t_{i}^{\text {out }} k$ is equal to the end taxi time $t_{e}$, satisfying the following equation:

$$
\left\{\begin{array}{l}
t_{i}^{\text {in }} k=t_{0}, i=1 \\
t_{i}^{\text {in }} k=t_{i-1}^{\text {out }} k=t_{i} \quad, \quad 2 \leq j \leq e-1 . \\
t_{i}^{\text {out }} k=t_{e}, i=e-1
\end{array}\right.
$$

Taxi time window vector: the set of occupied time windows for each region of aircraft $k$ on the planned taxi path $L_{k z}$. The time window $t w_{i}(k)$ of aircraft $k$ occupying taxiing region $Q_{i}$ can be defined as the two tuples $t_{i}^{\text {in }} k$ and $t_{i}^{\text {out }} k$ :

$$
t w_{i}(k)=\left(t_{i}^{\text {in }} k, t_{i}^{\text {out }} k\right) .
$$

Therefore, the taxiing time window vector $L_{k z}$ of the path (k) can be defined by

$$
T W(k)=\left[t w_{1}(k), t w_{2}(k), \ldots, t w_{e-1}(k)\right] .
$$

\section{Aircraft Taxiing Conflict Detection and Resolution}

When there is a conflict in the planned trajectory time window, the aircraft chooses to wait or change its path to resolve the taxiing conflict based on comparing its priorities. Highpriority aircraft can pass the conflict area directly according to the original planned path; low-priority aircraft can slow down in advance to wait or use the second best preselected path (a shortest path that avoid the conflict area and reach the end point). At the same time, the wake interval time $\lambda$ needs to be added in the calculation of the waiting time to meet the wake interval criteria for aircraft taxiing at the surface.

4.1. Aircraft Taxiing Regulations and Priority Determination. To ensure safer and more efficient airport operations, surface aircraft should follow certain rules when taxiing [17]. 

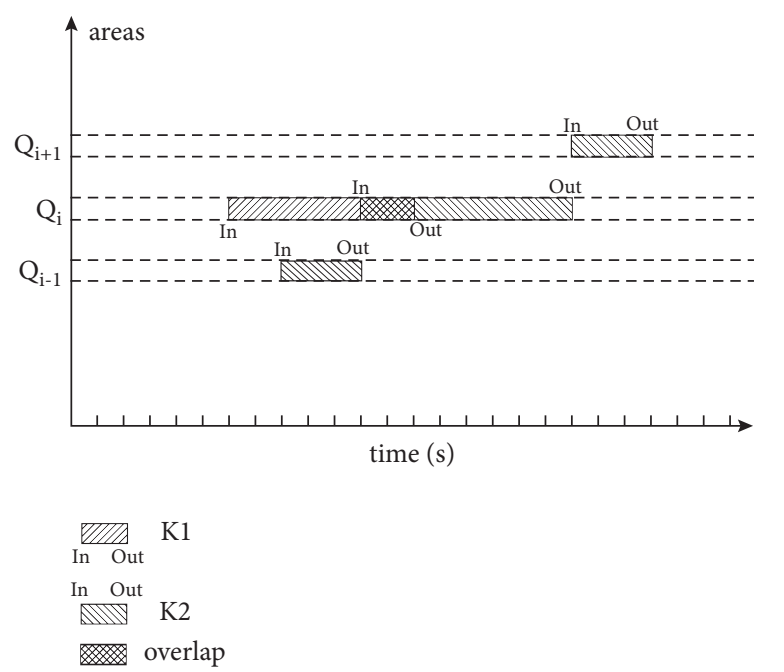

Figure 6: Time window for head-to-head conflict.

(1) The aircraft taxiing or towing on the surface must follow the prescribed or designated route by the surface controller; the taxi speed on the taxiway shall not exceed $50 \mathrm{~km} / \mathrm{h}$. After entering the passenger apron and apron, to ensure that it can be stopped at any time, the aircraft can only taxi at a slow speed.

(2) Two or more aircrafts will follow up taxiing. The rear aircraft shall not overtake the front aircraft. The distance between the rear aircraft and the front aircraft shall comply with the minimum separation standard specified by the wake interval: 100 meters from the tail of the taxiing light aircraft (light aircraft: aircraft with a maximum take-off weight of less than 7 tons); 200 meters from the tail of a medium-sized aircraft during taxiing (medium-sized aircraft: aircraft with a maximum take-off weight of 7-136 tons); and 300 meters from the tail of a heavy aircraft during taxi (heavy aircraft: maximum aircraft with a take-off weight greater than 136 tons).

The dynamic path optimization of the aircraft that taxi at the surface is based on their conflict relief priority. At present, the research of aircraft surface taxiing mainly adopts the first-come-first-served strategy, which is simple and practicable and reflects the principle of fairness. In view of this, the priority of flights during conflict detection and resolution is set as the priority of aircraft dispatch time (when the dispatch time is the same, the priority is determined by the peak of inbound and outbound flights).

\subsection{Aircraft Taxiing Head-to-Head Conflict Detection and} Resolution. Assuming that the moment when aircraft $k_{1}$ enters $Q_{i}$ is $t_{i}^{\text {in }}\left(k_{1}\right)$ and the moment when it leaves $Q_{i}$ is $t_{i}\left(k_{1}\right)$, the moment of aircraft $k_{2}$ entering $Q$ is $t_{i}^{\text {in }}\left(k_{2}\right)$ and the moment when it leaves $Q_{i}$ is $t_{i}^{\text {out }}\left(k_{2}\right)$. If there is a head-tohead conflict between aircraft $k_{1}$ and $k_{2}$, the taxiing area $Q_{i}$ is the head-to-head conflict section between aircraft $k_{1}$ and aircraft $k_{2}$, and then, the head-to-head conflict time window distribution is shown in Figure 6:
Therefore,

$$
\left\{\begin{array}{l}
t_{i}^{\text {in }}\left(k_{2}\right)<t_{i}^{\text {out }}\left(k_{1}\right), \\
t_{i}^{\text {out }}\left(k_{2}\right)>t_{i}^{\text {in }}\left(k_{1}\right) .
\end{array}\right.
$$

Assuming that the aircraft chooses to wait in place to complete the head-to-head conflict resolution, its waiting time $h$ can be found by

$$
h=t_{i}^{\text {out }}\left(k_{1}\right)-t_{i}^{\text {in }}\left(k_{2}\right)+\lambda .
$$

Then, the time of aircraft $k_{2}$ entering the taxiway area $Q_{i}$ can be updated as

$$
t_{i}^{\text {in }}\left(k_{2}\right)=t_{i-1}^{\text {out }}\left(k_{2}\right)+h .
$$

The updated aircraft taxiing occupancy time window is shown in Figure 7.

Assuming that the aircraft chooses to change the path to complete the conflict resolution, the aircraft is rescheduled for the suboptimal preselected path and the time window of the aircraft's taxiing area over the entire taxiway is updated.

4.3. Aircraft Taxiing Cross-Conflict Detection and Resolution. Assume that the moment of aircraft $k_{1}$ entering $Q_{i}$ is $t_{i}^{\text {in }}\left(k_{1}\right)$ and the moment of aircraft $k_{2}$ entering $Q_{i}$ is $t_{i}^{\text {in }}\left(k_{2}\right)$. If there is a cross-conflict between aircraft $k_{1}$ and $k_{2}$, the taxiing area $Q_{i}$ is the cross-conflict section of aircraft $k_{1}$ and aircraft $k_{2}$; then, the cross-conflict time window distribution is shown in Figure 8.

Then,

$$
\left|t_{i}^{\text {in }}\left(k_{2}\right)-t_{i}^{\text {in }}\left(k_{1}\right)\right|<\lambda
$$

Assuming that the aircraft chooses to wait in place to complete the cross-conflict resolution, its waiting time $h$ can be found by

$$
h=t_{i}^{\text {in }}\left(k_{2}\right)-t_{i}^{\text {in }}\left(k_{1}\right)+\lambda .
$$

Then, the time of aircraft $k_{2}$ entering the taxiway area $Q_{i}$ can be updated as

$$
t_{i}^{\text {in }}\left(k_{2}\right)=t_{i-1}^{\text {out }}\left(k_{2}\right)+h
$$

The updated taxiway occupancy time window is shown in Figure 9.

Assuming that the aircraft chooses to change the path to complete the conflict resolution, the aircraft is rescheduled for the suboptimal preselected path and the time window of the aircraft's taxiing area over the entire taxiway is updated.

4.4. Aircraft Taxiing Rear-End Conflict Detection and Resolution. Assuming that the moment when aircraft $k_{1}$ enters $Q_{i}$ is $t_{i}^{\text {in }}\left(k_{1}\right)$ and the moment when it leaves $Q_{i}$ is $t_{i}^{\text {out }}\left(k_{1}\right)$, the moment of aircraft $k_{2}$ entering $Q_{i}$ is $t_{i}^{\text {in }}\left(k_{2}\right)$ and the moment when it leaves $Q_{i}$ is $t_{i}\left(k_{2}\right)$. If there is a rear-end conflict between aircraft $k_{1}$ and $k_{2}$, the taxiing area $Q_{i}$ is the rear-end conflict section between aircraft $k_{1}$ and aircraft $k_{2}$, then the rear-end conflict time window. Distribution is shown in Figure 10. 

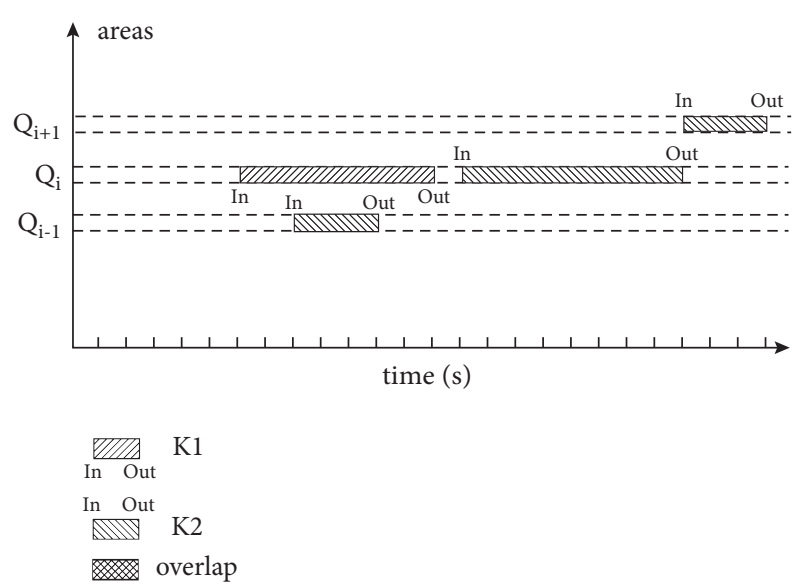

Figure 7: Update the time window for head-to-head conflict.
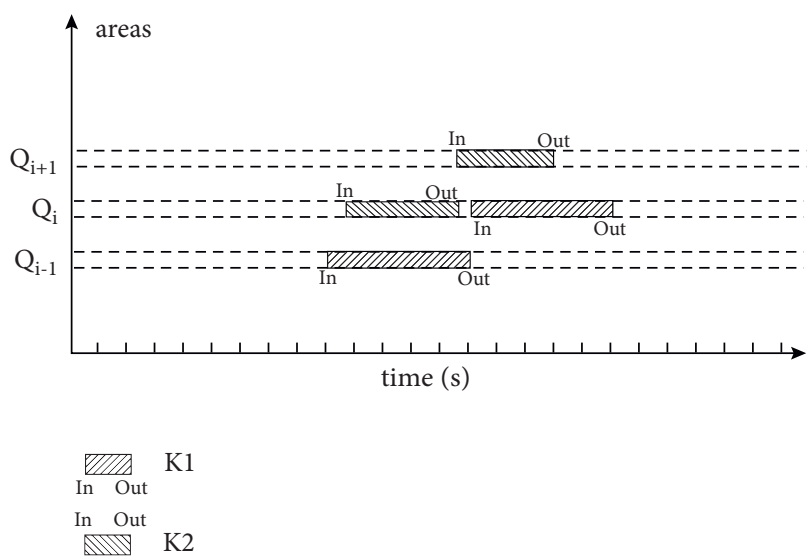

Figure 8: Time window for cross-conflict.
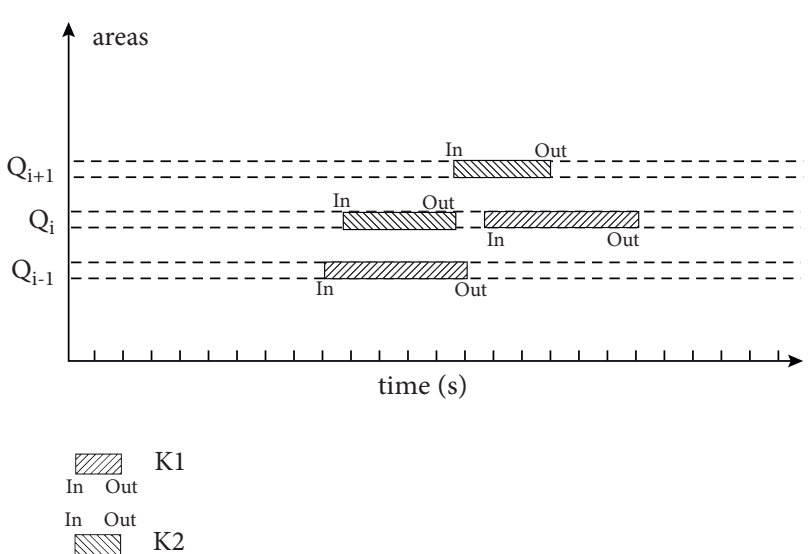

Figure 9: Update time window for cross-conflict.

Then,

$$
\left\{\begin{array}{l}
t_{i}^{\text {in }}\left(k_{2}\right) \geq t_{i}^{\text {in }}\left(k_{1}\right), \\
t_{i}^{\text {out }}\left(k_{2}\right) \leq t_{i}^{\text {out }}\left(k_{1}\right) .
\end{array}\right.
$$

Assuming that the aircraft chooses to wait in place to complete the rear-end conflict resolution, its waiting time $h$ can be found by
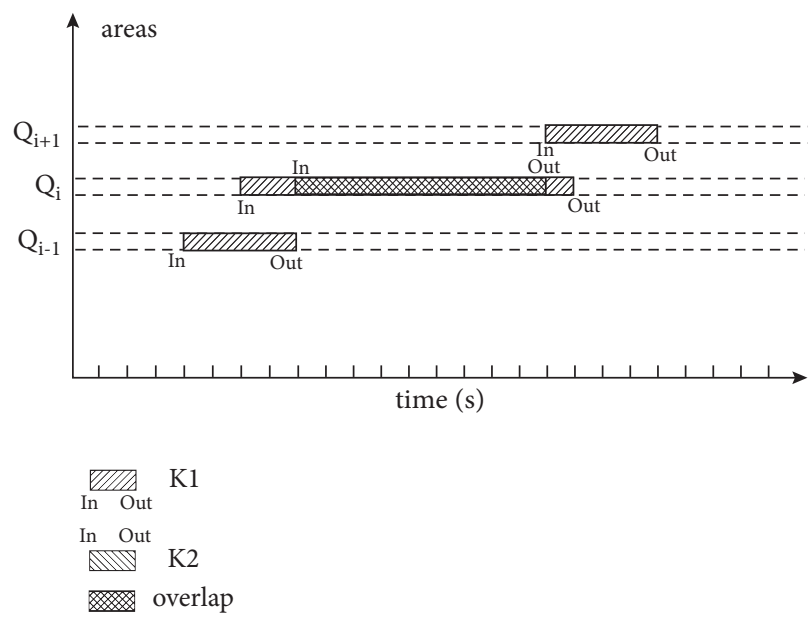

FIgure 10: Time window for rear-end conflict.

$$
h=t_{i}^{\text {out }}\left(k_{1}\right)-t_{i}^{\text {out }}\left(k_{2}\right)+\lambda .
$$

Then, the time of aircraft $k_{2}$ entering the taxiway area $Q_{i}$ can be updated as

$$
t_{i}^{\text {in }}\left(k_{2}\right)=t_{i-1}^{\text {out }}\left(k_{2}\right)+h
$$

The updated taxiway occupancy time window is shown in Figure 11.

Assuming that the aircraft chooses to change the path to complete the conflict resolution, the aircraft is rescheduled for the suboptimal preselected path, and the time window of the aircraft's taxiing area over the entire taxiway is updated.

4.5. Plane Taxiing Path with Aircraft Taxiing after the Conflict Is Resolved. If there are $n$ aircrafts operating at the airport surface, the time delay of taxiing aircraft $k$ conflict with other aircrafts is defined as $\sigma_{k}$ :

$$
\sigma_{k}=\sum h
$$

The objective of the dynamic path planning method is to minimize the total taxiing time of the surface operating aircraft by making the low-priority aircraft wait or change their paths to deconflict. Thus, its objective function is as follows:

$$
\begin{aligned}
& Z=t_{e}-t_{0}+\sigma_{k}, \\
& t_{0} \geq \text { ETOP, } \\
& t_{e} \leq \text { ETOD, }
\end{aligned}
$$

where $t_{0}$ is the time when the aircraft enters the starting taxiing section and $t_{e}$ is the time when the aircraft leaves the ending taxiing section. Equation (18) is the moment when the aircraft starts taxiing not earlier than the expected launch time, and equation (19) is the moment when the aircraft ends taxiing not later than the expected departure time ETOD. 

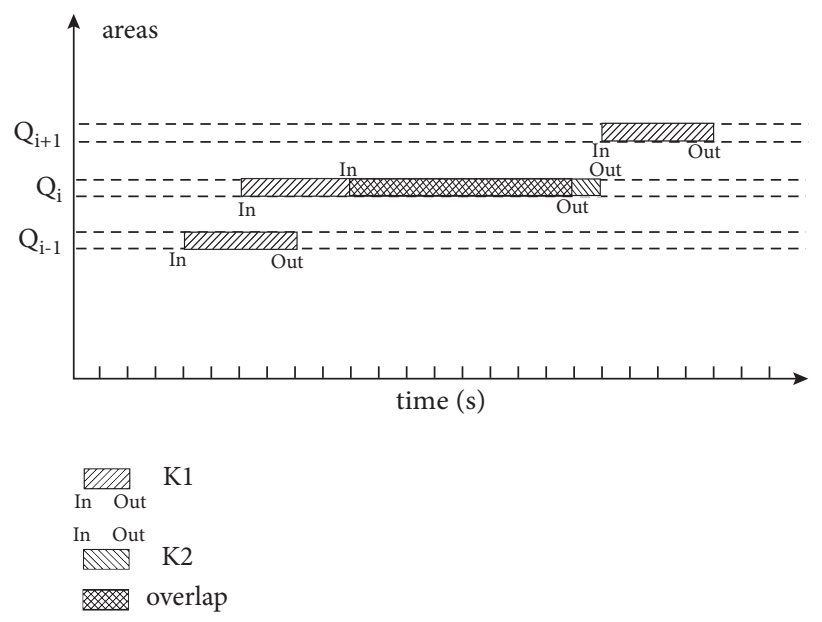

Figure 11: Update time window for rear-end conflict.

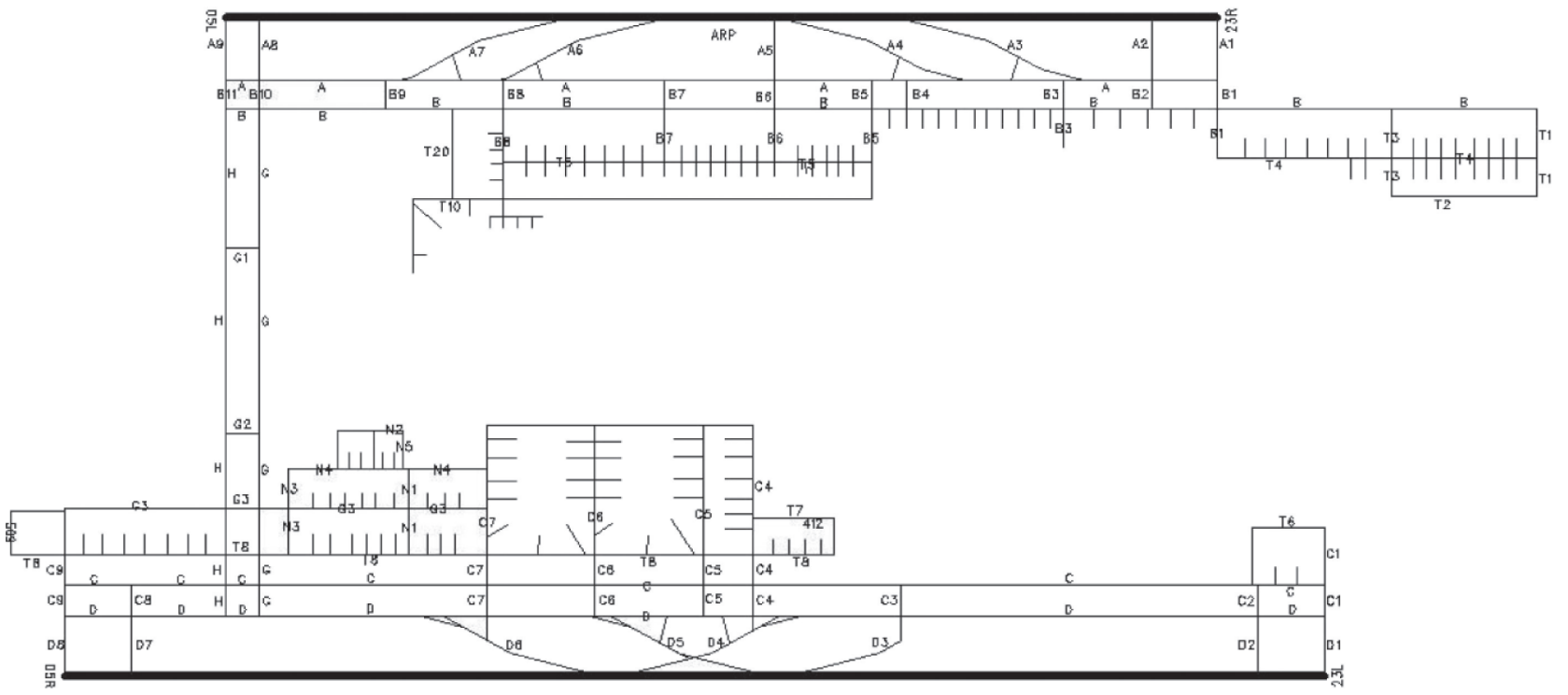

Figure 12: Xi'an Xianyang airport structure simplified diagram.

TABLe 1: Flight taxi schedule.

\begin{tabular}{lccc}
\hline Flight number & Arrival/departure & Starting point & Terminal point \\
\hline NS8264 & Arrival & 6 & 165 \\
HU7577 & Departure & 166 & 137 \\
MU4405 & Arrival & 154 & 167 \\
MU5569 & Departure & 168 & 1 \\
HU7817 & Departure & 169 & 1 \\
MU2158 & Arrival & 7 & 170 \\
\hline
\end{tabular}

\section{Example Application}

Take Xi'an Xianyang International Airport as an example. Here, the model and improved $A *$ algorithm are used to calculate the aircraft planning taxiing path. The airport has two parallel runways and three terminals. The airport structure simplified diagram is shown in Figure 12.
Taking into account the particularity of the epidemic, the flight data of Xi' an Xianyang Airport from 15:16:00 to 15:28:20 on May $2^{\text {nd }}, 2019$, is used for simulation calculation. The taxi schedule of flights is given in Table 1. Assume the surface taxi speed of the aircraft: $5 \mathrm{~m} / \mathrm{s}$ for the straight section and $4 \mathrm{~m} / \mathrm{s}$ for the turning section. The number of preselected paths $P$ of the aircraft is set to 3 , and the algorithm will plan out 3 preselected taxi paths with the shortest distance for each aircraft. 


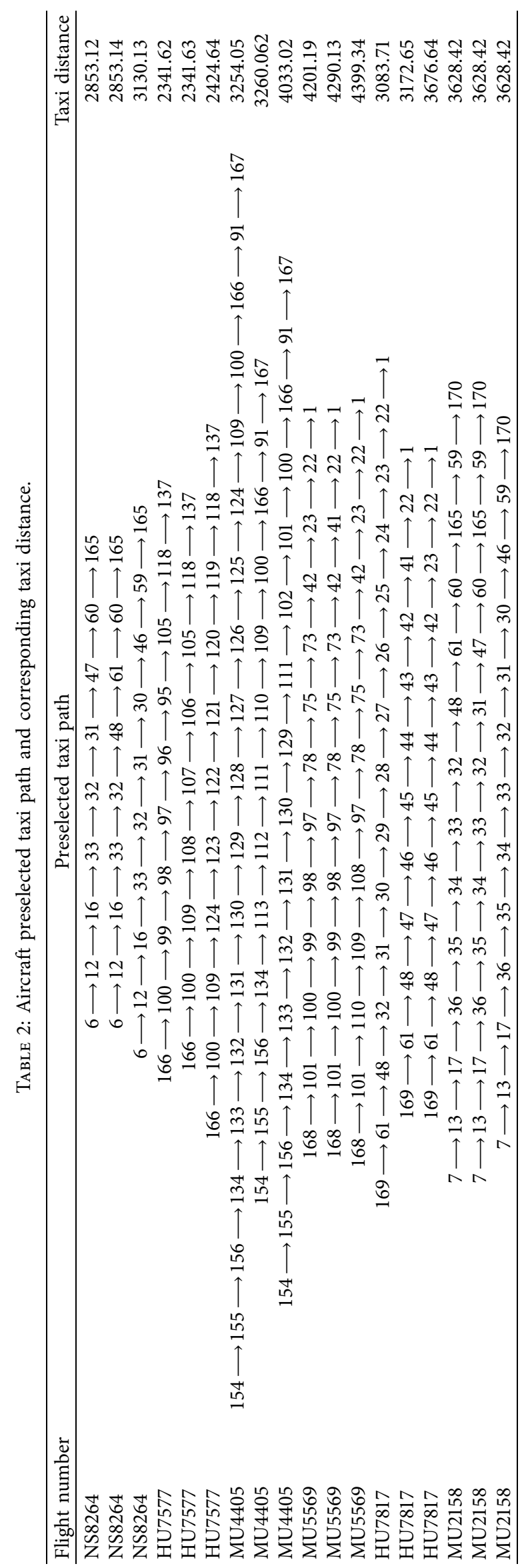




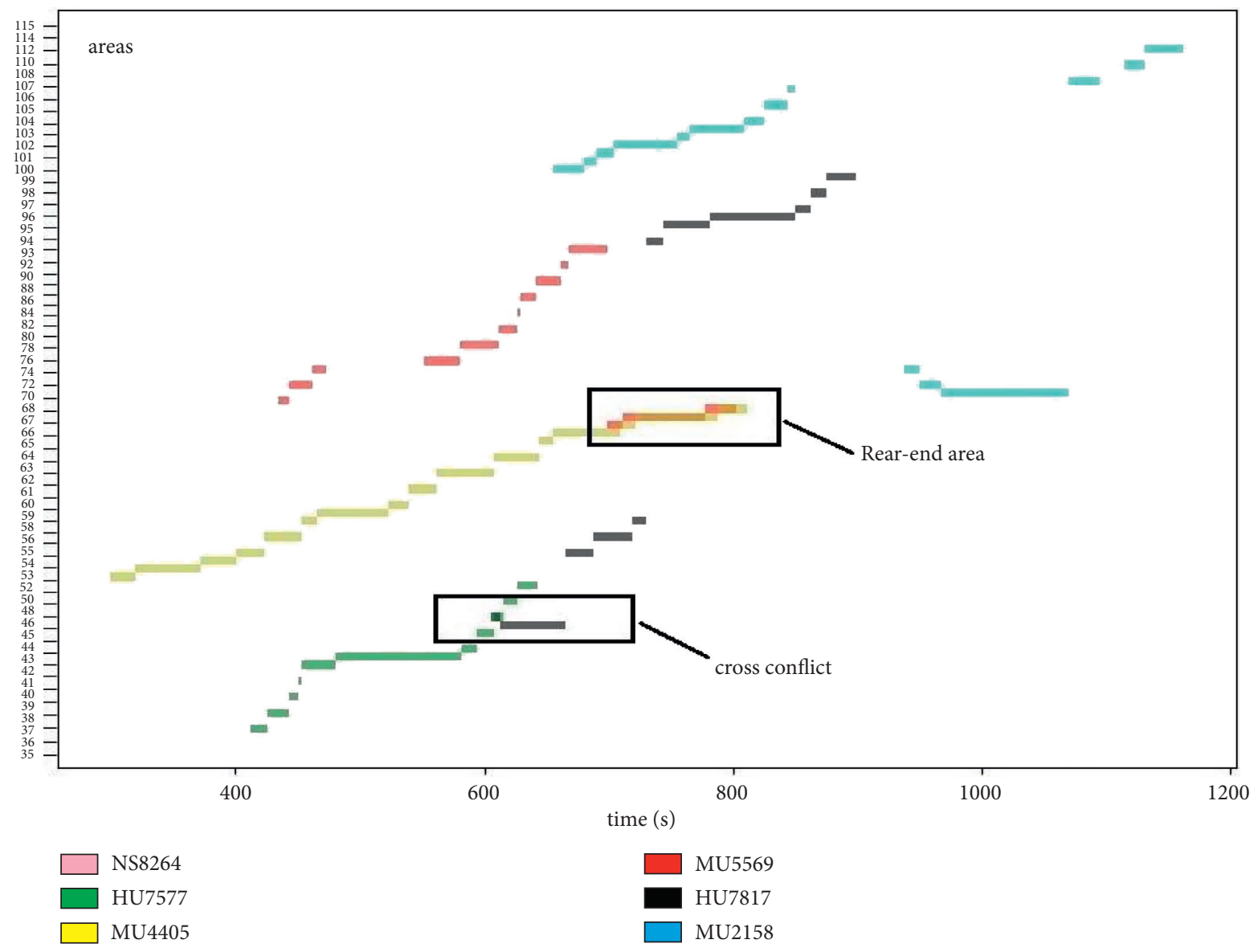

FIGURE 13: The area occupied the time window of the preselected route of the aircraft.

The calculation results of the three preselected taxi paths and their corresponding taxi distances for the six aircraft are given in Table 2 .

To further analyze the aircraft taxi path planning method after conflict resolution, select the preselected path with the shortest taxi distance in the preselected trajectory, calculate the time window occupied by the taxiway, perform conflict detection and relief, and finally generate the conflict-free and shortest taxi path. The taxiway structure model here covers the runway, parking stands, and main taxiway areas of Xi' an Xianyang Airport, including 218 areas. The time window occupied by the taxiway area of the aircraft during the study period is shown in Figure 13. The abscissa in the figure represents the taxi time, the ordinate represents the area number of the taxiing road segment, and the rectangle is the time window distribution of the preselected taxiing path of the aircraft in the area of each road segment.

From the figure, if taxiing along the preselected path, aircraft HU7577 and HU7817 will have a rear-end collision in area 48, and aircraft MU4405 and MU5569 will have a cross-collision in area 66. According to the priority regulations, adjust the taxi paths of the low-priority aircrafts HU7817 and MU5569. After adjustment, the taxi paths of the two aircrafts are shown in Figures 14(a) and 14(b).

From the figure, the initial planned trajectories of aircrafts MU5569 and HU7817 are both changed. Since the total taxiing time after waiting is less than that after changing the path, aircraft MU5569 enters area 67 after waiting in area 66 and continues to taxi on the initial planned path. Aircraft GS7657 enters area 46 after waiting in area 48 and continues to taxi on the initial planned path.

In addition, the planned paths of the other four aircrafts should not be disturbed by the deconfliction measures. The time change before and after the aircraft dynamic planning reaches the target point is given in Table 3, Due to conflict resolution, the time of aircrafts MU5569 and HU7817 reaching the target point increases, while the rest of the aircraft not affected remain unchanged, so that the conflict resolved does not affect the operation of other taxiing.

The waiting time, total taxiing time, total taxiing distance, and the number of conflicts are compared based on static taxiing path and dynamic path planning, respectively. As given in Table 3, in the results of the static taxiing path, the aircraft is requested to taxiing according to the shortest taxiing path without considering the conflicts caused to other aircrafts at the surface in the actual operation. In this example, there are two conflict points, and the dynamic path planning model finally plans a conflict-free taxiing path with the shortest taxiing time for the six aircraft in the study period.

Compare the waiting time, total taxi time, total taxi distance, and number of conflicts before and after conflict 


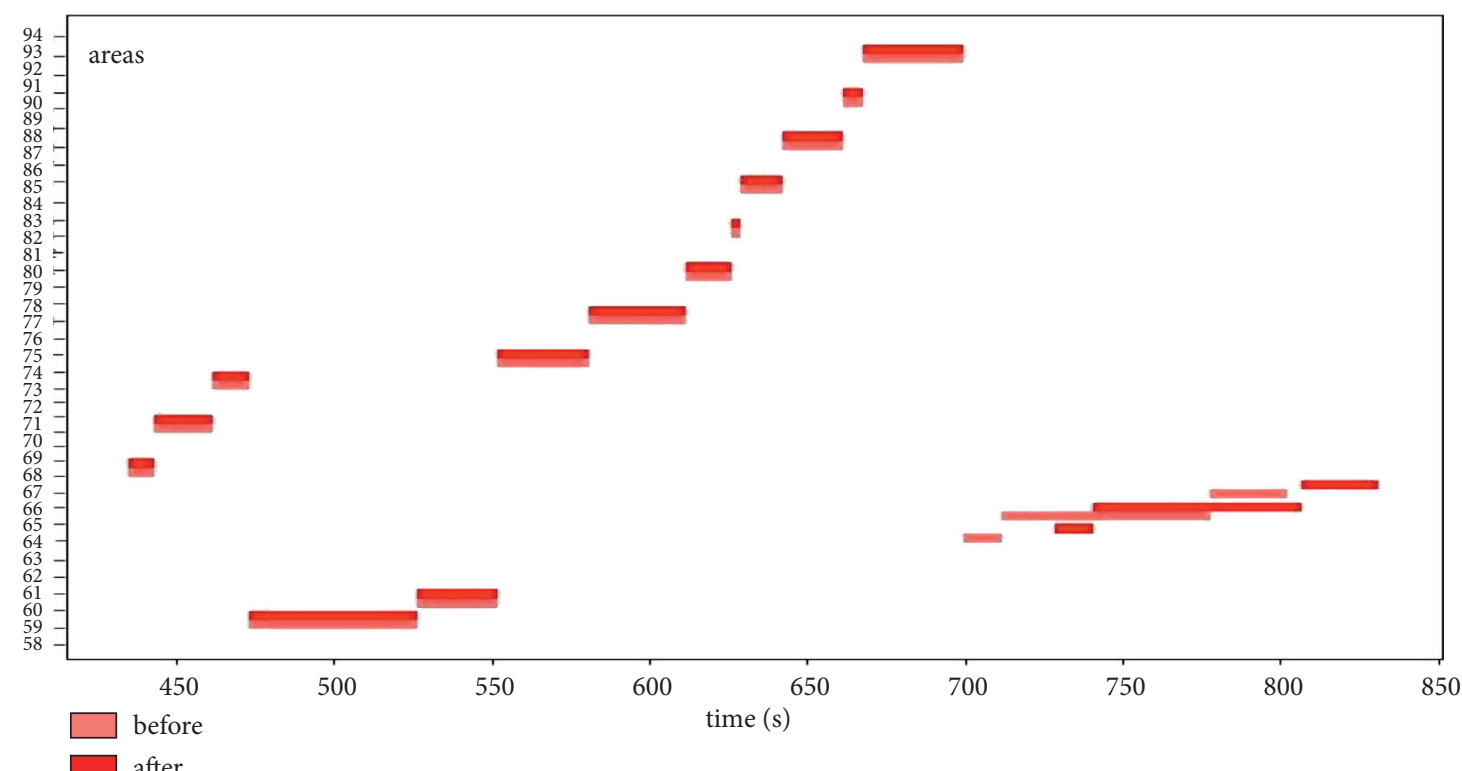

(a)

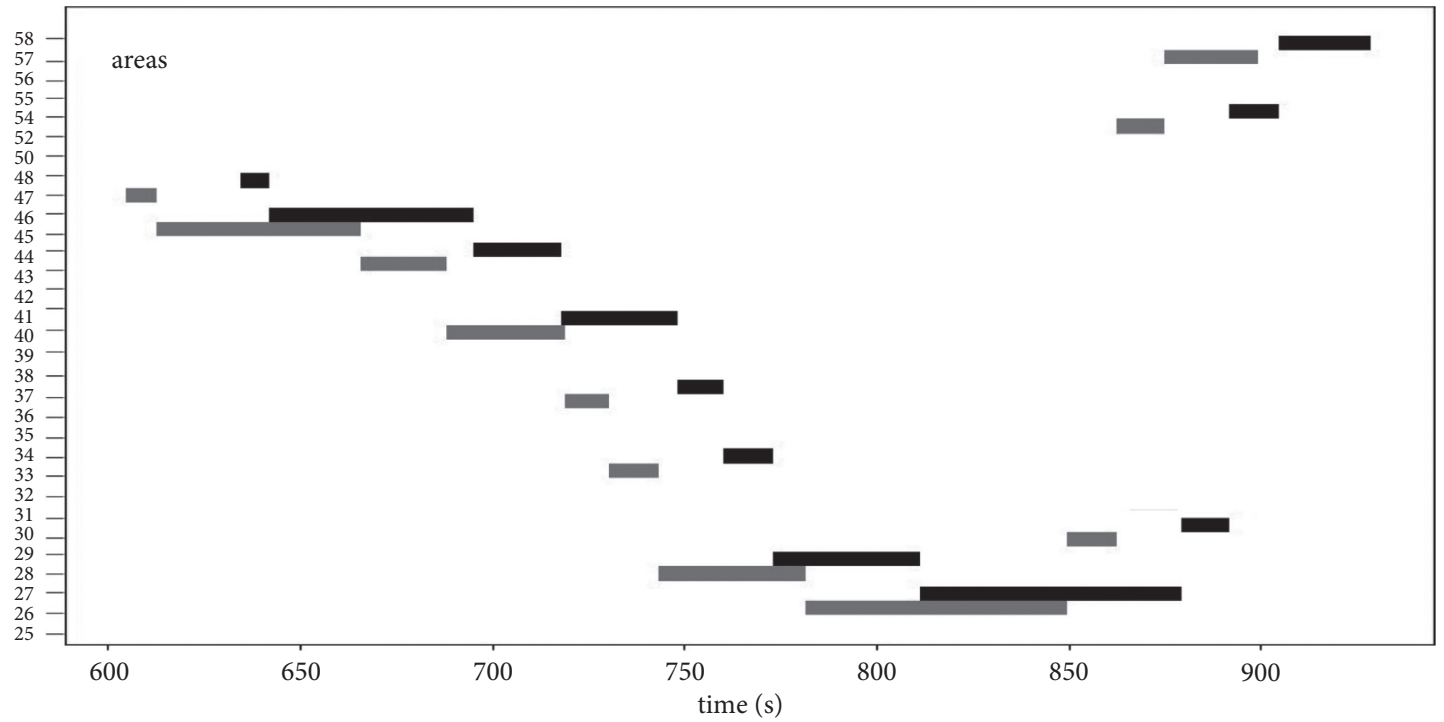

before

after

(b)

Figure 14: (a) Aircraft MU5569 area occupation time window before and after conflict resolution. (b) Aircraft HU7817 area occupation time window before and after conflict resolution.

Table 3: Changes in taxi time before and after the aircraft taxi path planning (unit: second).

\begin{tabular}{lccc}
\hline Aircraft & Initial taxi time & Taxi time after conflict resolution & Amount of change \\
\hline NS8264 & 427.83 & 427.83 & 0 \\
HU7577 & 294.21 & 294.21 & 0 \\
MU4405 & 467.51 & 467.51 & 0 \\
MU5569 & 511.98 & 541.57 & 29.59 \\
HU7817 & 367.28 & 396.43 & 29.15 \\
MU2158 & 506.64 & 506.64 & 0 \\
\hline
\end{tabular}


TABLE 4: Comparison of the results of taxi path planning before and after conflict resolution.

\begin{tabular}{lcccc}
\hline & Waiting time $(\mathrm{s})$ & Total taxi time $(\mathrm{s})$ & Total taxi distance $(\mathrm{m})$ & Number of conflicts \\
\hline Preselected taxi path & 0 & 2634.18 & 19225.52 & 2 \\
Taxi path after conflict resolved & 58.74 & 2575.44 & 19225.52 & 0 \\
\hline
\end{tabular}

resolution. As given in Table 4, in the preselected taxi path results, the aircraft is requested to taxi along the shortest taxi path, without considering the actual operation. Conflict is caused by other aircrafts on the scene. In this example, there are two conflict points. After the conflict is resolved, the time window model is used to finally plan a conflict-free and shortest taxi path for the six aircraft in the study period.

\section{Conclusion}

Through the study of the aircraft surface taxiing path, the airport surface structure model was established, the aircraft surface taxiing path planning model was constructed, and the improved $A *$ algorithm was used to solve the $P$ preselected paths with the shortest total taxi distance of the aircraft. According to the calculation results of the preselected path, the shortest taxi path is selected to calculate the time window occupied by the aircraft on the taxiing section, and the conflict of the time window occupied by the taxiing area is detected. According to the taxiing rules of the aircraft, the selection is based on the priority of comparison, wait or change the route to resolve the taxi conflict, generate a conflict-free taxi route for the aircraft, and update the preselected route time window. The calculation results of the calculation examples show that the use of the conflict resolution method in this study can further reduce the total taxi time of the aircraft operating on the surface, significantly reduce the number of aircraft conflicts, and ensure the safety of airport operations. In the research process, the $A *$ algorithm was used to calculate the shortest taxi path of the 6 flights. Although the $A *$ algorithm is more accurate, the calculation efficiency is low. Therefore, it is not easy to use the $A *$ algorithm when the number of flights is large, and a more appropriate algorithm should be selected for the solution. At the same time, it is assumed here that the taxi speed of the 6 flights is 5 meters per second in the straight section and the taxi speed in the turning section is 4 meters per second. When the number of flights is large, the taxi speed of the flight in the straight section and the turning section will be different. The deficiencies of these considerations also need to be improved in the follow-up research.

\section{Data Availability}

The data used to support the findings of this study are included within the supplementary information files. The supplementary information file contains the raw data for the calculation (the three XLS data files) and the program in the Python environment. (1) File "attribute.xls:" data of aircraft type, take-off weight, engine type and engine number, rated power, fuel flow rate (7\%), and fuel flow rate (30\%) of the six flights selected. (2) File "distance.xls:" the length from note 1 (the first column data) to note 22 (the second column data) is
13.335 meters (the third column data). (3) File "fixed_data.xls:" the sheet of node coordinate is the note coordinate from 1 to 170 . The sheet of turning is turning radius (the fourth column) determined by three points (the points in the first column, the points in the second column, and the points in the third column). The sheet of output is output data of the six flight. (4) File "Aerodrome Visualization:" the glide path and conflict resolution calculation program in Python environment.

\section{Conflicts of Interest}

The authors declare that they have no conflicts of interest.

\section{Acknowledgments}

This study was supported by Civil Aviation University of China, Civil Aviation Flight Wide Area Surveillance, and Safety Control Key Laboratory Fund (202008) and Fundamental Scientific Research Business Expenses for Central Universities of Civil Aviation University (3122020050).

\section{Supplementary Materials}

The supplementary information file contains the raw data for the calculation (the three XLS data files) and the program in the Python environment. (1) File "attribute.xls:" data of aircraft type, take-off weight, engine type and engine number, rated power, fuel flow rate $(7 \%)$, and fuel flow rate $(30 \%)$ of the six flights selected. (2) File "distance.xls:" the length from note 1 (the first column data) to note 22 (the second column data) is 13.335 meters (The third column data). (3) File "fixed_data.xls:" the sheet of node coordinate is the note coordinate from 1 to 170 . The sheet of turning is turning radius (the fourth column) determined by three points (the points in the first column, the points in the second column, the points in the third column). The sheet of output is output data of the six flights. (Supplementary Materials)

\section{References}

[1] Civil Aviation Administration of China, 2018 Civil Aviation Airport Production Statistics Bulletin, Civil Aviation Administration of China, Beijing, China, 2018.

[2] Civil Aviation Administration of China, 2018 National Civil Aviation Flight Operation Efficiency Report, Civil Aviation Administration of China, Beijing, China, 2018.

[3] Y. Wang, J. Zhu, and S. Ma, "Combination prediction of flight delays based on support vector regression and linear regression," Journal of Wuhan University of Technology, vol. 43, no. 3, pp. 426-431, 2019.

[4] X. Cheng, "Research on surface resource joint scheduling based on aircraft taxi time control," Master's Thesis, Nanjing 
University of Aeronautics and Astronautics, Nanjing, China, 2019.

[5] J. Chen, M. Weiszer, G. Locatelli et al., "Toward a more realistic, cost-effective, and greener ground movement through active routing: a multiobjective shortest path approach," IEEE Transactions on Intelligent Transportation Systems, vol. 17, no. 12 , pp. $3524-3540,2016$.

[6] N. K. Wickramasinghe, M. Brown, S. Fukushima, and Y. Fukuda, "Optimization-based performance assessment on 4D trajectory-based operations with track data," Air Traffic Management and Systems II, Springer, Berlin, Germany, 2017.

[7] T. Zhang, M. Ding, H. Zuo et al., "An online speed profile generation approach for efficient airport ground movement," Transportation Research Part C: Emerging Technologies, vol. 93, pp. 256-272, 2018.

[8] G. Yang, "Short-term and long-term hybrid igorithm for 4D trajectory prediction," Master Thesis, Civil Aviation University of China, Tianjin, China, 2016.

[9] Z. Wang, J. Xiong, and H. Chen, "A research on aircraft taxiing speed and fuel consumption," Journal of Wuhan University of Technology, vol. 41, no. 3, pp. 391-394, 2017.

[10] P. Podgrski and J. Skorupski, "Aircraft taxi route choice in case of conflict points existence," in Proceedings of the International Conference on Transport Systems Telematics, Springer International Publishing, Katowice-Ustroń, Poland, March 2016.

[11] N. Li, Y. Sun, J. Yu, J.-C. Li, H.-F. Zhang, and S. Tsai, “An empirical study on low emission taxiing path optimization of aircrafts on airport surfaces from the perspective of reducing carbon emissions," Energies, vol. 12, no. 9, p. 1649, 2019.

[12] X. Wang, "Research on taxiing route planning for aircraft based on surface hotspots," Master Thesis, Civil Aviation Flight University of China, Tianjin, China, 2015.

[13] N. Li, Q. Zhao, and X. Xu, "Researchon taxing optimization for aircraft based on improved $A *$ algorithm," Computer Simulation, vol. 29, no. 7, pp. 88-92, 2012.

[14] M. Yao, "Research on key techniques of aircraft sueface trajectory prediction and path planning in airport," Master Thesis, University of Electronic Science and Technology of China, Chengdu, China, 2018.

[15] T. Zhang, M. Ding, and H. Zuo, "Airport ground movement disruption recovery via mixed-integer programming," Journal of Beijing University of Aeronautics and Astronautics, vol. 44, no. 11, pp. 113-120, 2018.

[16] T. Zhang, H. Zuo, B. Wang, L. Zeng, and Z. Sun, "Zone control based aircraft ground movement trajectory optimization model," Systems Engineering and Electronics, vol. 38, no. 1, pp. 136-141, 2016.

[17] H. Chen, "Research on optimization technology of aircraft taxiing path in airport," Master Thesis, Nanjing University of Aeronautics and Astronautics, Nanjing, China, 2015. 